\title{
Performance Analysis of Gradual Multi-pulse Pulse-Position Modulation in Deep-Space Optical Communications
}

\author{
Hossam Selmy, Hossam M. H. Shalaby, and Zen Kawasaki
}

\begin{abstract}
A gradual multi-pulse pulse-position modulation (gradual MPPM) scheme is proposed as a new modulation scheme to improve both the performance and bandwidthutilization efficiency of the conventional optical multi-pulse pulse-position modulation (MPPM) scheme in deep-space optical communications. Whereas in the conventional MPPM scheme, a fixed number of optical pulses is transmitted in every signal block, a variable number of pulses are transmitted in the proposed scheme. Information is represented by different combinations of the positions of these pulses. The transmission characteristics, bandwidth utilization, and power requirements for the proposed scheme are studied in this paper. Several performance measures are derived and compared with those of the conventional MPPM scheme in deep-space optical communications. Our results reveal that, at the same average power, the proposed gradual MPPM scheme achieves much lower levels of symbol-error rates than those of the ordinary MPPM scheme, whereas, at the same peak power levels, the ordinary MPPM outperforms the gradual scheme. Also, in terms of bandwidth-utilization efficiency, the proposed modulation scheme achieves higher efficiency than the ordinary MPPM by allowing many more symbols to be transmitted per frame.
\end{abstract}

Index Terms-Deep-space optical communications; Gradual multi-pulse pulse-position modulation (gradual MPPM); Multipulse pulse-position modulation (MPPM); Symbol-error rate.

\section{INTRODUCTION}

$\mathbf{O}$ ne of the most popular modulation techniques that is used in optical direct-detection channels is single-pulseposition modulation (PPM) [1,2]. The advantage of using PPM is to locate the transmitted power efficiently in order to reduce the symbol-error rate (SER). Although increasing the number of frame slots reduces the SER, it further reduces the bandwidth-utilization efficiency and increases the synchronization errors. To overcome these drawbacks, multi-pulse pulse-position modulation (MPPM) has been

Manuscript received February 22, 2012; revised August 31, 2012; accepted September 3, 2012; published September 28, 2012 (Doc. ID 163468).

Hossam Selmy (e-mail: hossamselmy@yahoo.com) and Zen Kawasaki are with the Department of Electronics and Communications Engineering, Egypt-Japan University of Science and Technology (E-JUST), Alexandria 21934, Egypt.

Hossam M. H. Shalaby is with the Department of Electronics and Communications Engineering, Egypt-Japan University of Science and Technology (E-JUST), Alexandria 21934, Egypt, on leave from the Electrical Engineering Department, Alexandria University, Alexandria 21544, Egypt.

Digital Object Identifier 10.1364/JOCN.4.000812 proposed [3]. In MPPM, instead of transmitting a single optical pulse per frame, several pulses are allowed for transmission in order to increase their combinations per frame. Thus, a noticeable enhancement in bandwidth-utilization efficiency is achieved. Several performance comparisons between conventional single-pulse PPM and multi-pulse PPM have been made based on different criteria and under various transmission conditions [3-6]. The results indicate that the portion of bandwidth reduction achieved by MPPM is a function of the number of optical pulses per frame. Also, using this scheme, the transmission bandwidth could be reduced to about half that of conventional optical PPM at the same information rate.

However, at the same information rate and allowing different bandwidth utilizations, PPM outperforms MPPM in terms of bit-error rates (BERs). Clearly, this performance gap is reduced when a large number of slots per frame is implemented. Thus, different modulation formats are preferable under different transmission constraints imposed in deep-space communications, such as peak transmission power, average transmission power, and bandwidth utilization.

In this paper, we propose a new modulation scheme that improves both the SER and the bandwidth-utilization efficiency for the ordinary MPPM. In the proposed modulation scheme, which is called gradual multi-pulse pulse-position modulation (gradual $n$-pulse MPPM), we try to increase the number of transmitted symbols per frame while maintaining a reasonably small number of slots per frame. In addition, we derive several performance measures for our proposed system and compare them to those of the conventional MPPM scheme.

The rest of the paper is organized as follows: In Section II, we develop a general system model for the gradual $n$-pulse MPPM scheme and its variants. Also, the differences between the proposed gradual scheme and the ordinary scheme are stated. In Section III, we present the maximum-likelihood decoding (optimal decoding) for the proposed scheme on direct-detected optical signals with the presence of background radiation. In Section IV, we derive performance measures in terms of the SER under both the average transmission power constraint and the peak transmission power constraint. The performance comparisons and numerical results are presented in Section V. Finally, the conclusion is given in Section VI. 


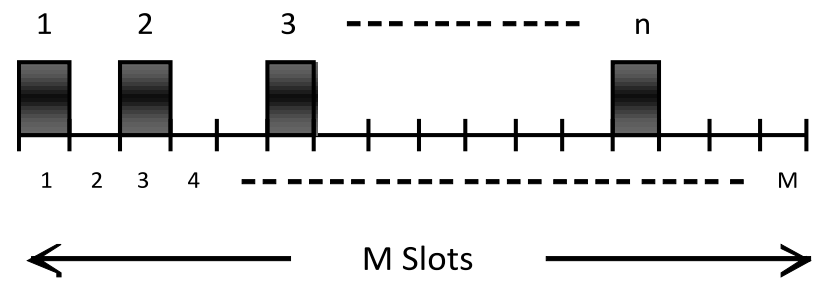

Fig. 1. Frame structure in ordinary MPPM.

\section{Proposed System Model AND CHARACTERISTICS}

In ordinary single-pulse PPM with a frame size of $M$ slots, the transmitted frames contain only one optical pulse that results in transmission of $\log _{2} M$ bits per frame. On the other hand, in ordinary multi-pulse pulse-position modulation ( $n$ pulse MPPM), the transmitted frames contain $n$ optical pulses, resulting in the transmission of $\log _{2}\left(\begin{array}{c}M \\ n\end{array}\right)$ bits per frame. Clearly, in order to increase this number, the modulation constellations must be increased. To achieve that, we propose a gradual multi-pulse pulse-position modulation (gradual $n$-pulse MPPM) scheme. In this scheme, instead of transmitting a fixed number of optical pulses ( $n$ pulses) per frame, we allow the transmission of one or more pulses (up to $n$ pulses) per frame. The resultant number of transmitted symbols per frame in this case is $\sum_{i=1}^{n}\left(\begin{array}{c}M \\ i\end{array}\right)$ symbols. Clearly, this number is much larger than the number of symbols available with ordinary MPPM schemes, which is $\left(\begin{array}{l}M \\ n\end{array}\right)$ symbols. Thus, with the same frame size and the same maximum number of transmitted optical pulses, the proposed gradual $n$-pulse MPPM scheme achieves a higher transmission capacity than the ordinary $n$-pulse MPPM scheme. Furthermore, in the proposed modulation scheme, the maximum number of transmitted optical pulses per frame $n_{G}$ is allowed to increase largely up to the value of $M$ in contrast to ordinary MPPM, where the value of the maximum number of transmitted optical pulses per frame $n_{M}$ is limited to $M / 2$. This valuable increase in the number of transmitted optical pulses per frame causes the bandwidth-utilization efficiency, achieved by the proposed scheme, to increase notably and reach nearly $100 \%$. Thus, another additional and important advantage arises for the proposed scheme.

The frame structures of both ordinary MPPM and the proposed gradual scheme are illustrated in Figs. 1 and 2, respectively. However, to clarify the idea of the proposed modulation scheme, we consider a numerical example in which the transmission frame consists of 8 slots. In ordinary single-pulse PPM, the number of available symbols for transmission is 8 symbols, producing a transmission of 3 bits per frame. For a 2-pulse 8-PPM scheme, there are $\left(\begin{array}{l}8 \\ 2\end{array}\right)=28$ symbols, resulting in the transmission of 4.8 bits per frame. Using a gradual 2-pulse 8-PPM scheme, both single and double optical pulses are allowed for transmission, resulting in a total of $8+28=36$ symbols and a transmission of 5.16 bits per frame. An increase of about $10 \%$ is obtained in the information rate. The maximum transmission utilization achievable by the MPPM scheme with a frame size of 8 slots occurs when $n_{M}=4$. In this case, the number of available symbols is 70 symbols,
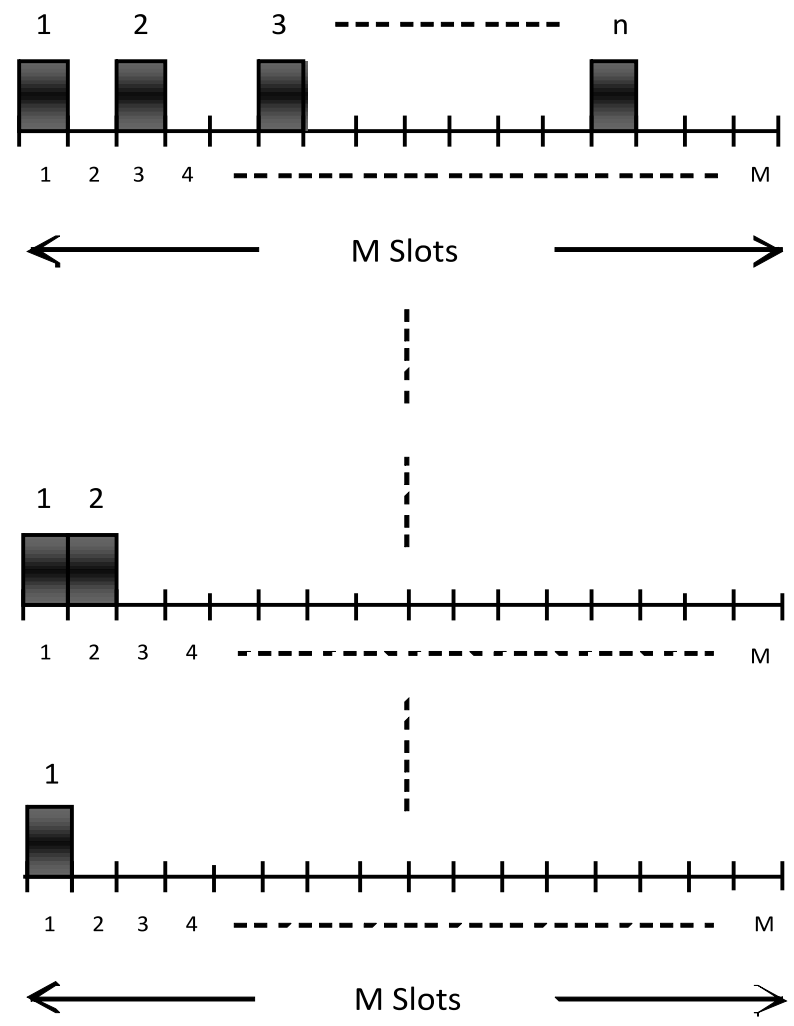

Fig. 2. Frame structure in gradual MPPM.

resulting in a transmission of 6.12 bits per frame. For the gradual 4-pulse 8-PPM case, the number of available symbols is $8+28+56+70=162$ symbols, thus achieving more than double the available symbols of ordinary 4-pulse 8-PPM.

Generally, in an ordinary $n$-pulse MPPM scheme, the number of transmitted bits per frame is $\log _{2}\left(\begin{array}{c}M \\ n_{M}\end{array}\right)$ (bits/frame), whereas in the gradual $n$-pulse MPPM scheme, this number is $\log _{2} \sum_{i=1}^{n_{G}}\left(\begin{array}{c}M \\ i\end{array}\right)$. Therefore, at the same values for $n_{M}$ and $n_{G}$, the information rate ratio is given by

$$
\text { Information rate ratio }=\frac{\log _{2} \sum_{i=1}^{n}\left(\begin{array}{c}
M \\
i
\end{array}\right)}{\log _{2}\left(\begin{array}{c}
M \\
n
\end{array}\right)} .
$$

In Fig. 3, we plot the information rate ratio for the case of $M=16$. As expected, the ratio increases by increasing the maximum number of optical pulses per frame, allowing more symbols to be available for transmission. Also, as stated before, the bandwidth-utilization efficiency increases significantly when using the proposed scheme. The bandwidth-utilization efficiency for the ordinary $n$-pulse MPPM scheme is given by

$$
U_{M}=\frac{\log _{2}\left(\begin{array}{c}
M \\
n_{M}
\end{array}\right)}{M},
$$

whereas the bandwidth-utilization efficiency for the gradual $n$-pulse MPPM scheme is given by 


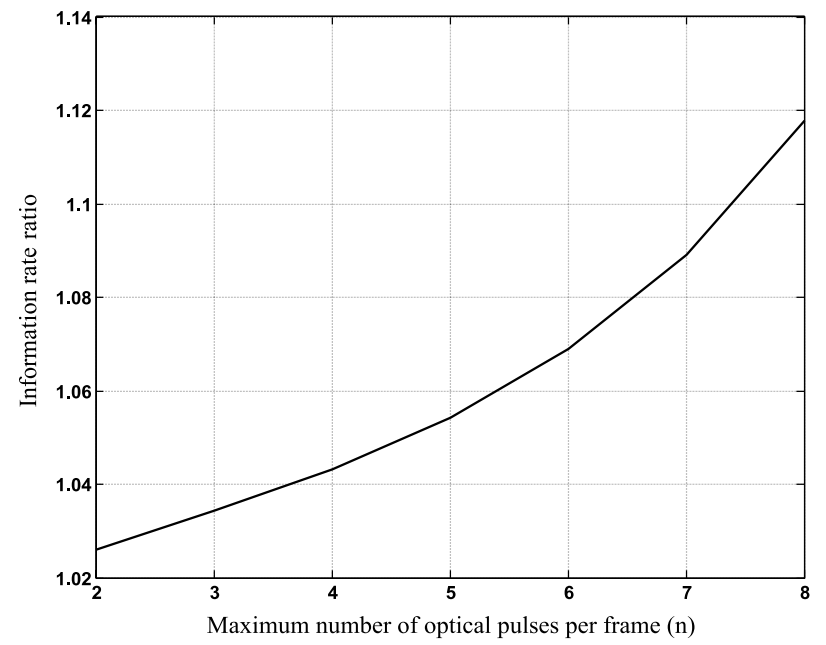

Fig. 3. Information rate ratio for $M=16$.

$$
U_{G}=\frac{\log _{2} \sum_{i=1}^{n_{G}}\left(\begin{array}{c}
M \\
i
\end{array}\right)}{M} .
$$

The bandwidth-utilization efficiencies for both schemes are compared at a fixed frame size of 16 slots. The comparison is carried out at different values of $n$, as shown in Fig. 4 . Obviously, the figure indicates that the maximum achievable efficiency for the $n$-pulse 16-MPPM scheme is about $85 \%$ and it occurs at $n=8$ pulses, whereas for the gradual $n$-pulse 16-PPM scheme, the utilization could approach 1 at $n=10$ pulses. Thus, the proposed modulation scheme is strongly intended to be used for applications that require efficient bandwidth utilizations.

\section{MaXimum-Likelihood Decoding}

Here, we aim at obtaining the optimal symbol decoding algorithm that minimizes the SER for the proposed gradual $n$-pulse MPPM scheme. The channel model for deep-space optical communications is considered in the analysis. In this channel, there is no dispersion, multipath, or atmospheric turbulence and only background radiation is assumed. Other optical channels could be considered by testing the appropriate channel model. However, to clarify the main aspects of the proposed modulation scheme and facilitate comparisons with well-known results, we consider a direct-detected discrete memoryless optical channel with Poisson distributions for both signal and background optical radiation. That is, a shot-noise-limited model for a photodetector with zero dark current and unity gain is assumed [7]. Furthermore, the detector thermal noise is neglected compared with high levels of background optical radiation. More accurate models exist for particular detectors, e.g., the Webb or Gaussian model for avalanche photodiodes (APDs) [8] and the Polya model for photomultiplier tubes (PMTs) [9], and they can be used to provide more accurate system modeling. However, much of the analysis here could be extended in a straightforward manner to these models.

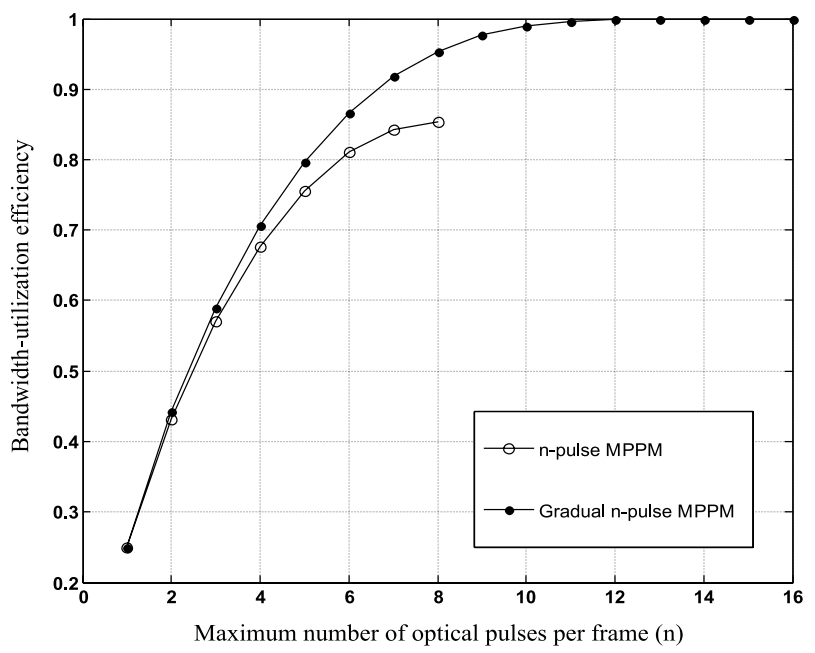

Fig. 4. Bandwidth-utilization efficiency.

The detected photon-counts associated with the received frame slots are summarized in the received $M$-component photon-count vector $\mathbf{k}=\left(k_{1}, k_{2}, k_{3}, \ldots, k_{n}, \ldots, k_{M}\right)$. Clearly, the probability of each count component is Poisson distributed with an average value of $K_{b}=\lambda_{b} \tau$ ( $\lambda_{b}$ denotes the background radiation intensity and $\tau$ is the slot time) when only background radiation is observed and $\lambda_{z i} \tau+K_{b}$ when both signal and background radiation are present [7]. Here, the subscript $z$ refers to the transmitted symbol $Z$, the subscript $i \in$ $\{1,2, \ldots, M\}$ identifies the slot number within the count vector, and $\lambda_{z i}$ denotes the signal intensity in slot $i$ for transmitted symbol $Z$. Generally, $\lambda_{z i} \tau$ is abbreviated by $K_{s}$ for the slot that contains an optical pulse.

Since, the count components are independent of each other, the conditional probability of a count vector $\mathbf{k}$ given that a certain hypothetical symbol $X$ is transmitted can be expressed as a product of $M$ independent Poisson distributions as

$$
P(\mathbf{k} \mid X)=\prod_{i=1}^{M} \frac{\left(\lambda_{x i} \tau+K_{b}\right)^{k_{i}}}{k_{i} !} e^{-\left(\lambda_{x i} \tau+K_{b}\right)} .
$$

The maximum-likelihood decoding is achieved by evaluating the conditional probabilities for the detected count vector given all hypothetical transmitted symbols and selecting the symbol that results in the maximum conditional probability. To get such a symbol with the maximum conditional probability given a received count vector, we consider the two following cases:

Case A: Here we consider any two hypothetical symbols $X$ and $Y$, each containing $j$ ones ( $j$ signal slots), where $j<n$. The division of their maximum-likelihood probabilities can be written as

$$
\begin{aligned}
\frac{P(\mathbf{k} \mid X)}{P(\mathbf{k} \mid Y)} & =\frac{\prod_{i=1}^{M} \frac{\left(\lambda_{x i} \tau+K_{b}\right)^{k_{i}}}{k_{i} !} e^{-\left(\lambda_{x i} \tau+K_{b}\right)}}{\prod_{i=1}^{M} \frac{\left(\lambda_{y i} \tau+K_{b}\right)^{k_{i}}}{k_{i} !} e^{-\left(\lambda_{y i} \tau+K_{b}\right)}} \\
& =\prod_{i=1}^{M}\left(\frac{\frac{\lambda_{x i} \tau}{K_{b}}+1}{\frac{\lambda_{y i} \tau}{K_{b}}+1}\right)^{k_{i}},
\end{aligned}
$$


assuming that symbol $X$ has $j$ ones associated with the counts $k_{x_{1}}, k_{x_{2}}, \ldots, k_{x_{j}}$ and symbol $Y$ has $j$ ones associated with the counts $k_{y_{1}}, k_{y_{2}}, \ldots, k_{y_{j}}$. Also, by ordering the received count vector $\mathbf{k}$ in a descending order to get the modified vector $\mathbf{k}^{*}=$ $\left(k^{1}, k^{2}, \ldots, k^{n}, \ldots, k^{M}\right)$, we have

$$
\frac{P(\mathbf{k} \mid X)}{P(\mathbf{k} \mid Y)}=\left(\frac{K_{s}}{K_{b}}+1\right)^{k_{x_{1}}+k_{x_{2}}+\cdots+k_{x_{j}}-k_{y_{1}}-k_{y_{2}}-\cdots-k_{y_{j}}} .
$$

Clearly, if we select the symbol $X$ such that

$$
k_{x_{1}}+k_{x_{2}}+\cdots+k_{x_{j}}=k^{1}+k^{2}+\cdots+k^{j},
$$

then $P(\mathbf{k} \mid X)>P(\mathbf{k} \mid Y)$ for any symbol $Y$ that contains $j$ ones. To summarize this point, for all symbols that have $j$ ones, the conditional probability will be maximized for the symbol that has its ones in the positions of the largest $j$ counts in the received count vector $\mathbf{k}$.

Case B: Here, we consider any two hypothetical symbols $X$ and $Y$ that have identical slot distributions except for one slot named $l$, whereas symbol $X$ has one and symbol $Y$ has zero. Then

$$
\begin{aligned}
\frac{P(\mathbf{k} \mid X)}{P(\mathbf{k} \mid Y)} & =\frac{\prod_{i=1}^{M} \frac{\left(\lambda_{x i} \tau+K_{b}\right)^{k_{i}}}{k_{i} !} e^{-\left(\lambda_{x i} \tau+K_{b}\right)}}{\prod_{i=1}^{M} \frac{\left(\lambda_{y i} \tau+K_{b}\right)^{k_{i}}}{k_{i} !} e^{-\left(\lambda_{y i} \tau+K_{b}\right)}} \\
& =\left(1+\frac{K_{s}}{K_{b}}\right)^{k_{l}} e^{-K_{s}}
\end{aligned}
$$

Let us define the threshold $T h$ as

$$
T h=\frac{K_{s}}{\ln \left(1+\frac{K_{s}}{K_{b}}\right)} .
$$

Thus, $P(\mathbf{k} \mid X)>P(\mathbf{k} \mid Y)$ if $k_{l}>T h$.

Clearly, from the aforementioned cases we could set the following optimal decoding (maximum-likelihood decoding) algorithm for the gradual MPPM scheme.

\begin{tabular}{l} 
Algorithm Steps \\
\hline 1) Sort the received photon-counts in descending order. \\
2) Decode the first maximum photon-count as one. \\
3) For $i=2$ to $n$ \\
If (next maximum $\geq T h$ ) \\
Then: Decode it as one \\
Else: Decode it and the remaining counts as zeros and end \\
the algorithm.
\end{tabular}

4) Decode the remaining $M-n$ slots as zeros.

\section{PERFormance Evaluation}

In this section we aim at evaluating the SER for gradual $n$-pulse MPPM. We recall the calculation of SER for ordinary $n$-pulse MPPM as stated in [10]. In ordinary $n$-pulse MPPM with a memoryless channel, without loss of generality, we could assume that the signal slots of the transmitted symbol occupy the first $n$ slots of the frame. Then, the probability of decoding the received count vector $\mathbf{k}$ correctly is the probability that $\min \left(k_{1}, k_{2}, \ldots, c, k_{n}\right) \geq \max \left(k_{n+1}, \ldots, k_{M}\right)$. If the equality holds, the decoder chooses randomly among multiple decoding decisions and only one of them is correct. To compute this probability, let $p_{0}\left(k_{i}\right)$ and $p_{1}\left(k_{i}\right)$ denote the count probabilities of slot $i$ in the cases of non-signal and signal slots, respectively. Also, let $P_{0}\left(k_{i}\right)$ and $P_{1}\left(k_{i}\right)$ denote their cumulative distributions. Furthermore, assume that $k_{\max }=$ $\max \left(k_{n+1}, \ldots, k_{M}\right)$ with $l$ repetitions in the $M-n$ non-signal slots and $m$ repetitions in the $n$ signal slots, i.e., $1 \leq l \leq M-n$, $0 \leq m \leq n$. Clearly, for any values of $l$ and $m$, there are $\left(\begin{array}{c}l+m \\ m\end{array}\right) \stackrel{\text { def }}{=} \frac{1}{I(l, m)}$ different decoding decisions with only one of them being correct. Thus, the SER for $n$-pulse MPPM is given by [10]

$$
\begin{aligned}
\text { SER }= & 1-\sum_{k_{\max =0}}^{\infty} \sum_{l=1}^{M-n} \sum_{m=0}^{n} I(l, m)\left(\begin{array}{c}
M-n \\
l
\end{array}\right) p_{0}\left(k_{\max }\right)^{l} \\
& \times P_{0}\left(k_{\max }-1\right)^{M-n-l}\left(\begin{array}{l}
n \\
m
\end{array}\right) p_{1}\left(k_{\max }\right)^{m} \\
& \times\left(1-P_{1}\left(k_{\max }\right)\right)^{n-m}
\end{aligned}
$$

Next, we evaluate the SER for the gradual $n$-pulse MPPM for $n>1$. Clearly, the case $n=1$ (i.e., ordinary PPM) could not be considered as a special case of gradual MPPM, instead it may be considered as a special case of ordinary $n$-pulse MPPM with $n=1$. However, in the evaluation of the SER for gradual $n$-pulse MPPM, we first evaluate the probability to receive the transmitted symbols correctly, which is denoted by $p(c)$. The evaluation of $p(c)$ for gradual $n$-pulse MPPM must consider the following cases of transmitted symbols:

Case A: In this case, we compute the probability of correct transmission for symbols that contain only one signal slot. Considering one such symbol, which has its signal in the first slot, the probability to receive this symbol correctly is the probability that $k_{1} \geq \max \left(k_{2}, k_{3}, \ldots, k_{M}\right)$ and $\max \left(k_{2}, k_{3}, \ldots, k_{M}\right)<T h$. If the first equality holds, i.e., $k_{1}=$ $\max \left(k_{2}, k_{3}, \ldots, k_{M}\right)$, then there are multiple decoding decisions and only one of them is correct. To account for this, let $k_{\max }=\max \left(k_{2}, k_{3}, \ldots, k_{M}\right)$ and let $l$ denote the number of non-signal slots that have this maximum count. Here, the decoder randomly chooses one slot out of $l+1$ slots to be decoded as one and the rest are decoded as zeros. Thus, the probability to make a correct decision is $I(l, 1)=\frac{1}{l+1}$. Also, we have $\left(\begin{array}{c}M-1 \\ l\end{array}\right)$ similar ways corresponding to the number of possible combinations for the remaining $M-1$ slots. Summing all the combinations for values of $l$ from 1 to $M-1$ and considering the two events $m=0$ when $k_{1}>k_{\max }$ and $m=1$ when $k_{1}=k_{\max }$, we obtain the total probability to receive this symbol correctly as

$$
\begin{aligned}
p(c)_{A}= & \sum_{k_{\max }=0}^{T h-1} \sum_{l=1}^{M-1} \sum_{m=0}^{1} I(l, m)\left(\begin{array}{c}
M-1 \\
l
\end{array}\right) p_{0}\left(k_{\max }\right)^{l} \\
& \times P_{0}\left(k_{\max }-1\right)^{M-1-l} p_{1}\left(k_{\max }\right)^{m} \\
& \times\left(1-P_{1}\left(k_{\max }\right)\right)^{1-m} .
\end{aligned}
$$


Case B: In this case, we compute the probability of correct transmission for symbols that contain a number of signal slots $i$ that is greater than one and less than $n(1<i<n)$. Without loss of generality, we consider one such symbol, which has its pulses in the first $i$ slots and the remaining $M-i$ slots are non-signal slots. The decoding decision is made correctly for this symbol only if $\left(k_{1}, k_{2}, \ldots, k_{i} \geq T h>k_{i+1}, \ldots, k_{M}\right)$. Therefore, the probability to decode this symbol correctly is given as

$$
p(c)_{B i}=\left[1-P_{1}(T h-1)\right]^{i}\left[P_{0}(T h-1)\right]^{M-i} .
$$

Case $C$ : In this case, we compute the probability of correct transmission for the symbols that contain $n$ signal slots. Considering one such symbol that has its pulses in the first $n$ slots, the decoding decision is made correctly for this symbol only when $\min \left(k_{1}, k_{2}, \ldots, k_{n}\right) \geq \max \left(k_{n+1}, \ldots, k_{M}\right)$ and $\min \left(k_{1}, k_{2}, \ldots, k_{n}\right) \geq T h$. However, if the second equality holds, multiple decisions could be made and only one of them is correct. To consider these possibilities, let $k_{\min }=$ $\min \left(k_{1}, k_{2}, \ldots, k_{n}\right)$. Also, let $l$ and $m$ denote the number of signal slots and non-signal slots that have this minimum value, respectively, i.e., $0 \leq l \leq M-n$ and $1 \leq m \leq n$. Here, the decoder chooses randomly $m$ slots out of $l+m$ possible slots with the probability of correct selection equal to $I(l, m)=\frac{1}{\left(\begin{array}{c}l+m \\ l\end{array}\right)}$.

Also, there are $\left(\begin{array}{c}n \\ m\end{array}\right)\left(\begin{array}{c}M-n \\ l\end{array}\right)$ different ways corresponding to the number of possible combinations for each value of $l$ and $m$. Summing all the combinations for values of $m$ from 1 to $M-n$ and values of $l$ from 1 to $n$, we obtain the probability of correction decoding for this symbol as

$$
\begin{aligned}
p(c)_{c}= & \sum_{k_{\min }=T h}^{\infty} \sum_{l=0}^{M-n} \sum_{m=1}^{n} I(l, m)\left(\begin{array}{c}
M-n \\
l
\end{array}\right) \\
& \times P_{0}\left(k_{\min }-1\right)^{M-n-l}\left(\begin{array}{l}
n \\
m
\end{array}\right) p_{1}\left(k_{\min }\right)^{m} \\
& \times\left(1-P_{1}\left(k_{\min }\right)\right)^{n-m} .
\end{aligned}
$$

Finally, the total probability of correct symbol transmission for gradual $n$-pulse MPPM can be evaluated as the average probability of the correct decoding over all the transmitted symbols and thus the SER is given by

$$
\begin{aligned}
\text { SER }= & 1-p(c) \\
= & 1-\frac{1}{\sum_{j=1}^{n}\left(\begin{array}{c}
M \\
j
\end{array}\right)}\left[\left(\begin{array}{c}
M \\
1
\end{array}\right) p(c)_{A}\right. \\
& \left.+\sum_{i=2}^{n-1}\left(\begin{array}{c}
M \\
i
\end{array}\right) p(c)_{B i}+\left(\begin{array}{c}
M \\
n
\end{array}\right) p(c)_{C}\right] .
\end{aligned}
$$

\section{NumericAl Results}

In this section we compare the performance of the proposed gradual $n$-pulse MPPM to ordinary $n$-pulse MPPM in terms of the average SER, average received power, and peak received power. To clarify the comparison, we use the same frame size $M$ and the same slot duration $\tau$ for both schemes, resulting in the same frame rate. The evaluations are carried out for two values of the frame size, namely, $M=8$ and $M=16$. For the case $M=8$, in order to achieve nearly the same number of transmission symbols for both schemes, the compared schemes are chosen to be 4-pulse 8-PPM (with number of signal pulses per frame $n_{M}=4$ ) and gradual 3-pulse 8-PPM (with maximum number of signal slots per frame $n_{G}=3$ ). In this selection the number of available symbols in the 4-pulse 8-PPM scheme is 70 symbols, whereas in the gradual 3-pulse 8-PPM scheme, the number of the available symbols for transmission is 92 symbols. Clearly, gradual 3-pulse 8-PPM achieves much more transmission capacity than 4-pulse 8-PPM, which represents another advantage in their comparison. For the case $M=16$, we choose 8-pulse 16-PPM and gradual 6-pulse 16-PPM schemes to compare. Clearly, the numbers of symbols available for transmission per frame for these modulations are 12,870 and 14,892 symbols, respectively, which add an extra advantage to the gradual scheme.

The bit encoding for gradual MPPM could be achieved using the same encoding techniques of ordinary MPPM. However, there is no known efficient method of encoding MPPM symbols [11]. The simplest, but inefficient, encoding scheme is to encode each symbol by the integer number of bits resulting from taking the logarithm of the number of available symbols in the system. As an example of such encoding, consider the case of the 4-pulse 8-PPM scheme, each frame is encoded by 6 bits only while the remaining fraction of bits $(0.129$ bits $)$ is discarded. Clearly, in this case not all available symbols are used for transmission and there is an optimal bit-symbol mapping that results in the lowest BER. Another simple and more efficient encoding scheme is to create compound symbols by cascading enough MPPM symbols to ensure that the total number just exceeds a power of 2 and then encoding suitably larger blocks of information bits into this compound signal set [2]. The bit encoding and decoding for both the gradual and ordinary MPPM along with the transmitter and receiver complexity are not in the scope of this paper. However, they represent important design issues that could be considered in other research works.

Consequently, in our evaluations, the comparisons between gradual MPPM and ordinary MPPM are based on SER not BER because the exact relation between SER and BER for both schemes varies and depends on the implemented bit-symbol mapping codes.

The background radiation (noise photons) is considered in our evaluations by the mean of the average number of received background photons per slot $K_{b}$. The evaluations are performed at two noise levels, which are $K_{b}=1$ and $K_{b}=5$. On the other hand, in terms of the received optical power, the comparisons are performed under two constraints, namely, the average power constraint and the peak power constraint.

\section{A. Average Power Constraint}

The average power constraint is usually imposed in the case where the transmitter has limited power resources. That is, 


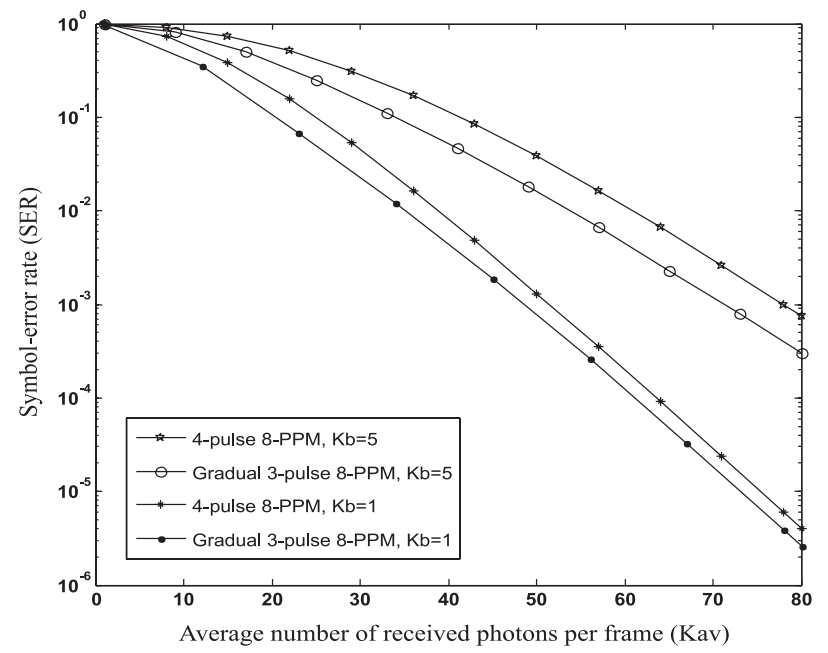

Fig. 5. Symbol-error rate versus average received photons per frame at $M=8$.

the transmitter has a bound on the number of emitted photons per transmitting frame. This case arises in free-space optical (FSO) systems working on solar energy and space optical communications between spacecraft. Clearly, the modulation scheme that achieves higher performance at the same average energy level is highly desired in this case.

To minimize the compared parameters, we fix the frame size for both schemes. Thus, the average power comparison can be replaced by the average number of received photons per frame $K_{a v}$. The relation between the average number of received photons per frame $K_{a v}$ and the signal slot photons $K_{s}$ are different for the two schemes.

For $n$-pulse MPPM the average number of received photons per symbol $K_{a v M}$ is given by

$$
K_{a v M}=K_{s M} \times n_{M}
$$

For gradual $n$-pulse MPPM the average number of received photons $K_{a v G}$ is given by

$$
K_{a v G}=\frac{K_{s G} \times \sum_{i=1}^{n_{G}} i \times\left(\begin{array}{c}
M \\
i
\end{array}\right)}{\sum_{i=1}^{n_{G}}\left(\begin{array}{c}
M \\
i
\end{array}\right)} .
$$

For the equal average power constraint we have $K_{a v M}=$ $K_{a v G}$. Figures 5 and 6 show the first group for comparison, where we evaluate the performance of the proposed gradual $n$-pulse MPPM scheme against the performance achieved by the $n$-pulse MPPM scheme in terms of received SER versus the average number of received photons per frame as given by Eqs. (10) and (14). The results for the case $M=8$ are shown in Fig. 5, which carries the comparison between two specific schemes: 4-pulse 8-PPM and gradual 3-pulse 8-PPM. Obviously, as mentioned before, the gradual scheme achieves higher bandwidth utilization than that of the ordinary scheme. Figure 5 indicates the out-performance of the

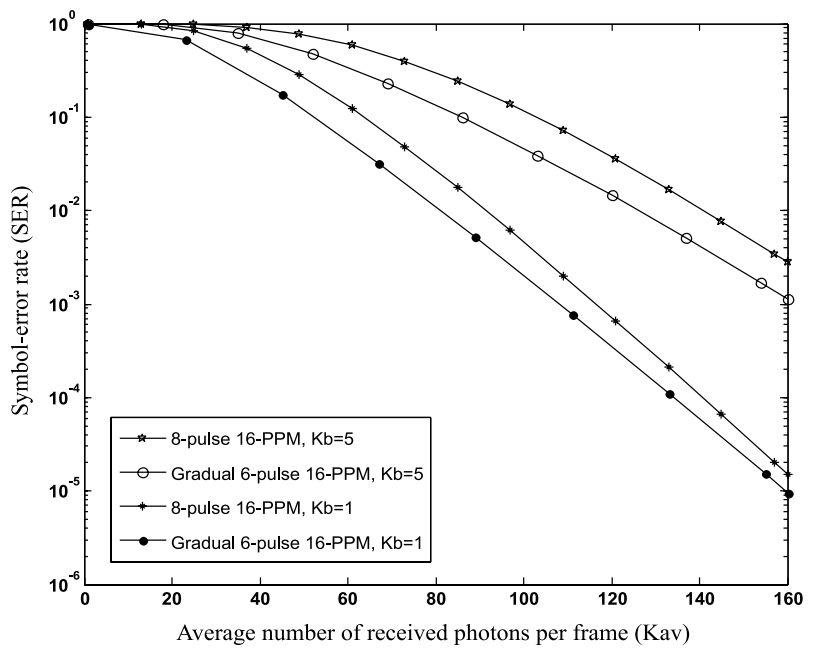

Fig. 6. Symbol-error rate versus average received photons per frame at $M=16$.

proposed schemes in achieving less SER at different values of average received photons per frame $K_{a v}$.

The comparisons are carried out at two values of the background noise $K_{b}=1$ and $K_{b}=5$. Also, the figure indicates better performance of the proposed scheme for greater background noise. Specifically, at $K_{a v}=40$ photons the gradual $n$-pulse MPPM achieves $6 \mathrm{~dB}$ reduction in SER at $K_{b}=$ 1 and a reduction of $8 \mathrm{~dB}$ at $K_{b}=5$. This large reduction in SER at higher $K_{b}$ levels makes the gradual scheme noise robust. For the case $M=16$ as indicated in Fig. 6, the comparison is carried out between two modulation schemes, 8-pulse 16-PPM and gradual 6-pulse 16-PPM. Again, this selection achieves nearly the same bandwidth utilization for both schemes with an advantage to the gradual scheme. The figure emphasizes the superior performance of the proposed gradual scheme over the ordinary scheme. Furthermore, this performance gap is increased by increasing the level of background noise. This could be interpreted as follows: At the same average number of received photons per frame $K_{a v}$, the pulse power in the gradual scheme is much higher than the pulse power in the ordinary scheme, which in turn causes the gradual scheme to perform much better in noisy channels.

The analysis of the achieved SER performance is explained further by Figs. 7 and 8 . These figures indicate the effect of the average number of received photons per frame $K_{a v}$ (which represents a metric for the average power) on the number of received photons in signal slot $K_{s}$ (which represents a metric for the peak power). Obviously, in the gradual schemes, the average number of signal slots per frame is much less than the number of signal slots per frame in ordinary MPPM schemes with the same frame sizes. This fact, along with transmitting the same average number of photons per frame $\left(K_{a v}\right)$, introduces a noticeable increase in the number of received photons per signal slot $\left(K_{s}\right)$ for the gradual schemes compared with that of the ordinary schemes. Consequently, with the same background noise levels, i.e., the same values of $K_{b}$, the detection of the gradual scheme symbols is less erroneous than that of the ordinary MPPM schemes. However, this performance enhancement comes with the price of a 


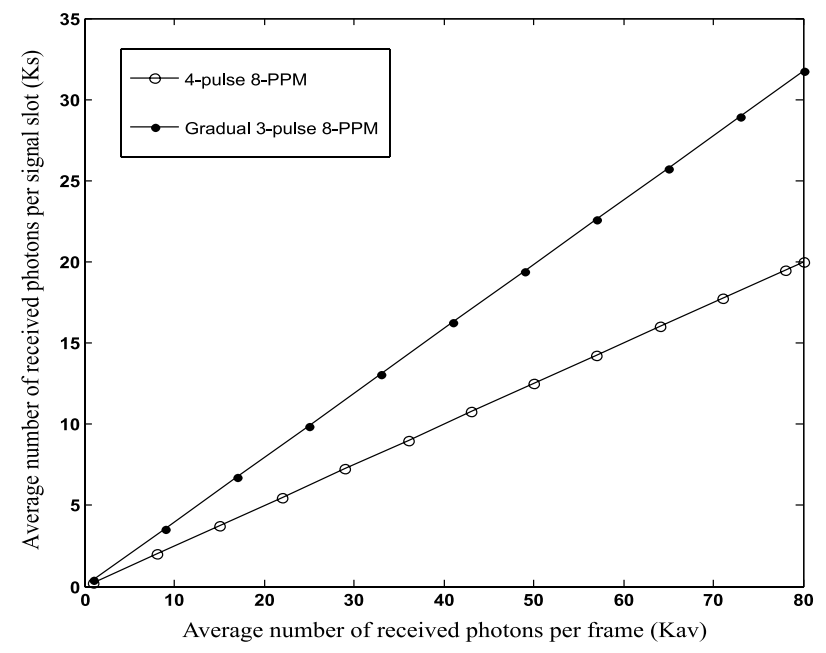

Fig. 7. Average number of photons per signal slot versus average received photons per frame at $M=8$.

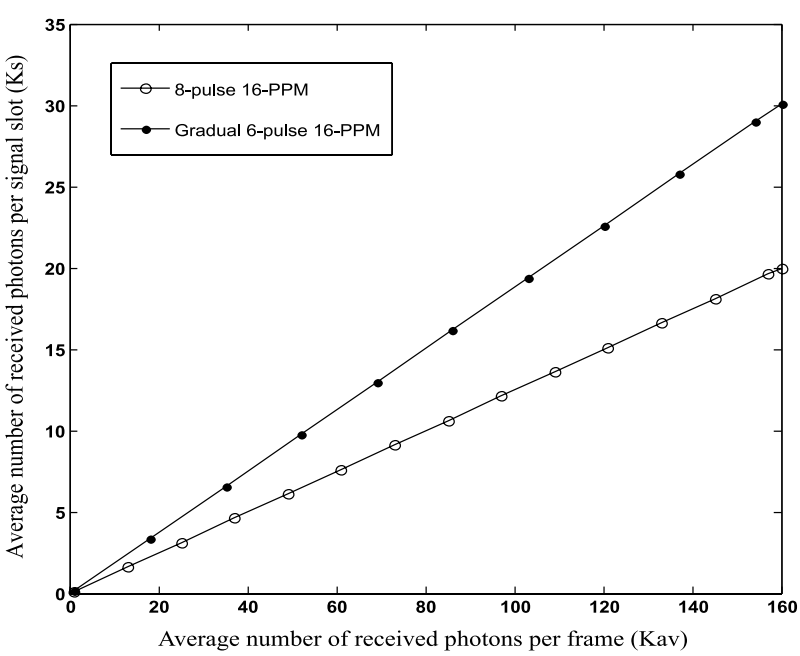

Fig. 8. Average number of photons per signal slot versus average received photons per frame at $M=16$.

noticeable increase in peak power levels, which may not be desirable for both transmitter amplifier saturation and safety regulations.

Numerically, as indicated in Figs. 7 and 8, for the same average received power, the gradual $n$-pulse MPPM schemes cause the number of received photons per signal slot $K_{s}$, and consequently the peak power, to increase linearly with about one and half times the rate of increase of the ordinary schemes. However, this rate of increase may limit the performance achieved by the gradual schemes to values lower than that achieved by the ordinary schemes in the case that the same maximum peak power level is used. Clearly, this case implies the peak power constraint, which is considered next.

Generally, the performance improvement achieved by gradual MPPM comes with additional complexities in the transmitter and receiver structure compared with PPM and ordinary MPPM. These additional complexities arise from

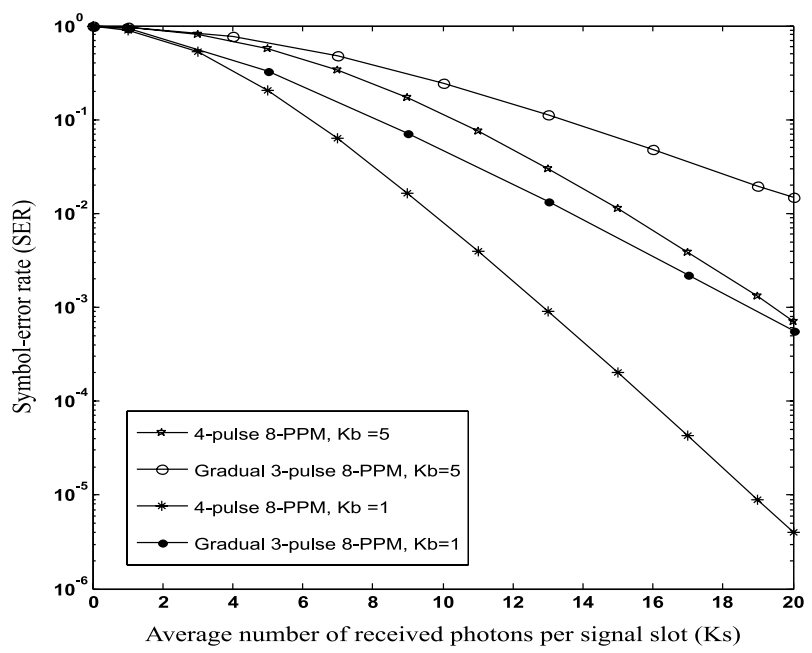

Fig. 9. Symbol-error rate versus average received photons per signal slot at $M=8$.

the need for periodical estimation of the threshold level on the receiver side along with the need to implement complex synchronization schemes to compensate for synchronization errors caused by false detected slots.

\section{B. Peak Power Constraint}

The peak power constraint is imposed in the cases where narrow-high-peak-power-transmitted pulses are required to overcome a high level of detected background noise. Precisely, this constraint implies that the transmitter has a bound on the number of emitted photons per slot. However, this bound is generally limited to the saturation level associated with the transmitter power amplifier. The implementation of such constraints is realized in deep-space communications and FSO systems working from a permanent electrical supply. Clearly, the modulation scheme that achieves higher performance at the same peak power level is highly desired in this case.

Here, with fixed frame size and slot duration, the peak power constraint means that the average number of received photons during each signal slot is the same for both the gradual and ordinary schemes. Precisely, this means that both $K_{s M}$ and $K_{s G}$ are equal. Also, the average numbers of received photons per frame for both schemes $K_{a v M}$ and $K_{a v G}$ are still given by Eqs. (15) and (16), respectively. The evaluations of SER under the peak power constraint are shown in Figs. 9 and 10 for cases $M=8$ and $M=16$, respectively. For the case $M=8$, we compare the performance achieved by 4-pulse 8-PPM and gradual 3-pulse 8-PPM schemes, whereas, for the case of $M=16$, we use 4-pulse 8-PPM and gradual 3-pulse 8-PPM schemes. The figures indicate that lower SER values are achieved by the ordinary schemes than by the gradual schemes at the same $K_{s}$ levels. Moreover, the performance gap between the two schemes is significantly increased by raising the level of $K_{s}$. Specifically, for the case of $M=8$ and $K_{b}=1$, doubling the value of $K_{s}$ from 10 to 20 photons per slot causes the performance gap to increase from $9 \mathrm{~dB}$ to $22 \mathrm{~dB}$. Furthermore, at any fixed value of $K_{s}$, this performance gap 


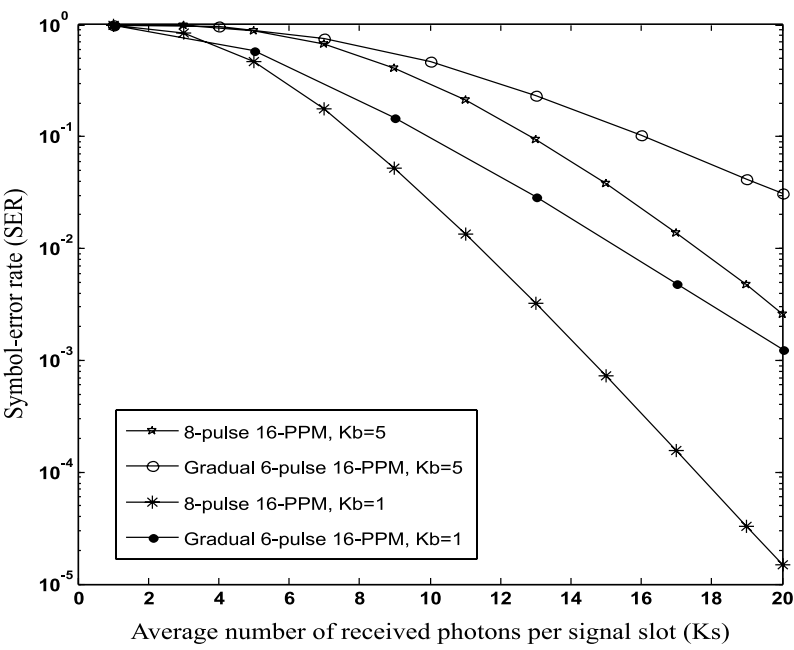

Fig. 10. Symbol-error rate versus average received photons per signal slot at $M=16$.

is decreased by increasing the background noise level $\left(K_{b}\right)$. This indicates a relative reduction in the performance gap between the ordinary and proposed schemes at higher levels of background noise.

Generally, this superior performance, achieved by the ordinary schemes, comes with the price of requiring a larger amount of average power than the gradual schemes. This is clearly shown in Figs. 11 and 12 for the cases of $M=8$ and $M=16$, respectively. As indicated by Fig. 11, the amount of average power required by the ordinary scheme is about one and half times the amount needed by the gradual scheme at the same transmission peak power level. Also, nearly the same ratio is required for the case of $M=16$ as shown in Fig. 12 . These results come from the fact that the average number of signal slots per frame for the gradual scheme is less than that of the number of signal slots for the ordinary scheme. Also, with the peak power constraint, all signal slots have the same amount of optical energy, resulting in higher average power levels for the ordinary schemes.

\section{CONCLUSion}

A new modulation scheme, gradual $n$-pulse MPPM, of the family of PPMs has been proposed for optical communications. The proposed scheme achieves much higher bandwidthutilization efficiency than that of the ordinary MPPM scheme. On a discrete memoryless channel, the maximum-likelihood decoding criteria for the proposed scheme were derived, resulting in a simple and fast decoding algorithm. The performance measure of the proposed scheme in terms of exact SER was obtained. Intensive numerical evaluations were performed to compare the performance achieved by the proposed gradual scheme with the performance of the ordinary MPPM scheme. The comparisons were carried out under both average power and peak power constraints at nearly the same transmission rates. The results indicate the superior performance of the proposed gradual scheme under average power constraints. In contrast, for the same peak power levels,

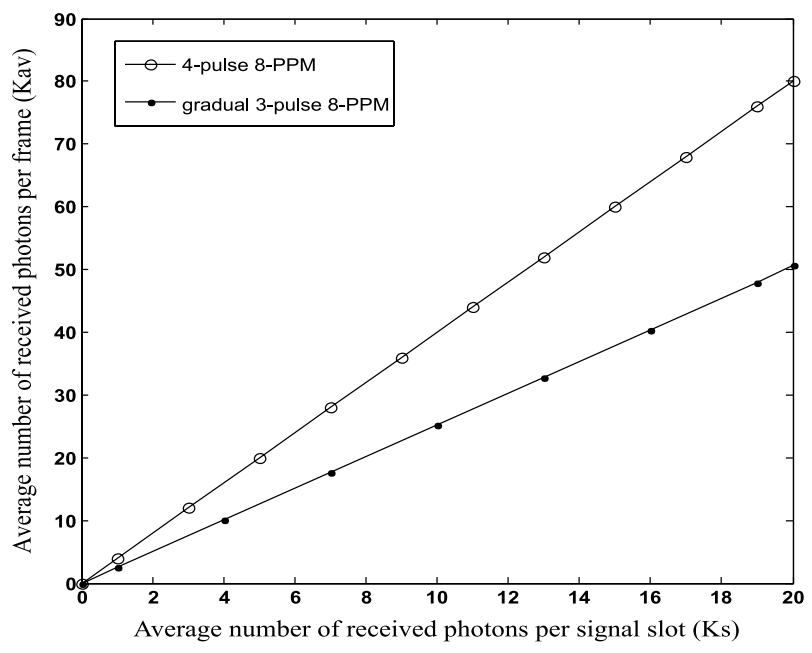

Fig. 11. Average number of received photons per frame versus average received photons per signal slot at $M=8$.

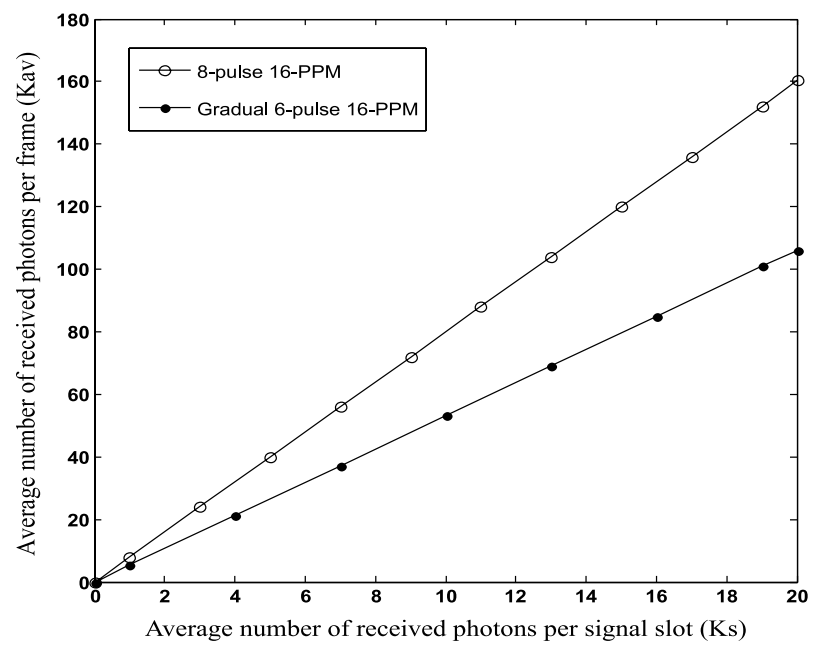

Fig. 12. Average number of received photons per frame versus average received photons per signal slot at $M=16$.

it was demonstrated that the ordinary $n$-pulse MPPM results in much better performance. Thus, no unique modulation scheme can fit all circumstances.

\section{ACKNOWLEDGMENTS}

The authors wish to thank the Ministry of Higher Education of Egypt for its grant for this research.

\section{REFERENCES}

[1] J. Singh and V. K. Jain, "Performance analysis of BPPM and $M$-ary PPM optical communication systems in atmospheric turbulence," IETE Tech. Rev., vol. 25, no. 4, pp. 146-153, July-Aug. 2008.

[2] M. Simon and V. Vilnrotter, "Performance analysis and tradeoff for dual-pulse PPM on optical communications channels with 
direct detection," IEEE Trans. Commun., vol. 52, no. 11, pp. 1969-1979, Nov. 2004.

[3] H. Sugiyama and K. Nosu, "MPPM: A method for improving the band utilization efficiency in optical PPM," J. Lightwave Technol., vol. 7, no. 3, pp. 465-471, Mar. 1989.

[4] H. Park, "Performance bound on multiple-pulse position modulation," Opt. Rev., vol. 10, no. 3, pp. 131-132, May 2003.

[5] M. F. Barsoum, B. Moision, M. Fitz, D. Divsalar, and J. Hamkins, "Iterative coded pulse-position-modulation for deep-space optical communications," in Information Theory Workshop, Sept. 2007, pp. 66-71.

[6] K. Sato, T. Ohtsuki, and I. Sasase, "Performance of coded multi-pulse PPM with imperfect slot synchronization in optical direct-detection channel," in Proc. IEEE Int. Communications Conf. (ICC), 2004, vol. 1, pp. 121-125.

[7] R. M. Gagliardi and S. Karp, Optical Communications. 2nd ed., John Wiley \& Sons, 1995.

[8] S. Dolinar, D. Divsalar, J. Hamkins, and F. Pollara, "Capacity of pulse-position modulation (PPM) on Gaussian and Webb channels," The Telecommunications and Mission Operations Progress Report 42-142, Apr.-June 2000, Jet Propulsion Laboratory, Pasadena, California, Aug. 15, 2000, pp. 1-31.

[9] A. Biswas and W. H. Farr, "Laboratory characterization and modeling of a near-infrared enhanced photomultiplier tube," The Interplanetary Network Progress Report 42-152, Oct.-Dec. 2002, Jet Propulsion Laboratory, Pasadena, California, Feb. 15, 2003, pp. 1-14.

[10] J. Hamkins and B. Moision, "Multipulse PPM on memoryless channels," in Int. Symp. on Information Theory (ISIT), Chicago, Illinois, June 2004.

[11] S. Liu and F. R. Kschischang, "Coding for MPPM-like systems," in 25th Biennial Symp. on Communications (QBSC 2010), Kingston, Ontario, Canada, May 12-14, 2010, pp. 365-368.

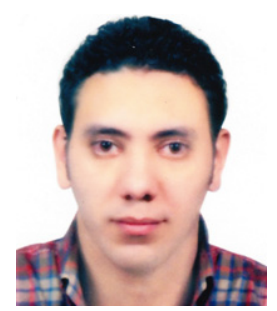

Hossam Selmy was born in Giza, Egypt, in 1979. He received the B.S. and M.S. degrees from Cairo University, Cairo, Egypt, in 2001 and 2007, respectively. He is currently pursuing the Ph.D. degree at the Graduate School of Engineering, Egypt-Japan University for Science and Technology (EJUST), Alexandria, Egypt. His research interests include advanced optical modulations and multiple access schemes for both optical fiber communications and free-space optical communications systems.

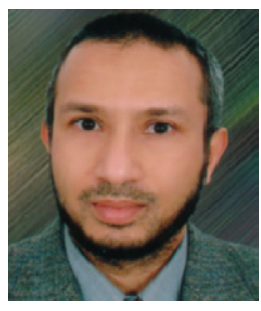

Hossam M. H. Shalaby (S'83-M'91-SM'99) was born in Giza, Egypt, in 1961. He received the B.S. and M.S. degrees from Alexandria University, Alexandria, Egypt, in 1983 and 1986, respectively, and the Ph.D. degree from the University of Maryland at College Park in 1991, all in electrical engineering. In 1991, he joined the Electrical Engineering Department, Alexandria University, and was promoted to Professor in 2001. Currently he is on leave from Alexandria University, where he is the chair of the Department of Electronics and Communications Engineering, School of Electronics, Communications, and Computer Engineering, Egypt-Japan University of Science and Technology (E-JUST), New Borg El-Arab City, Alexandria, Egypt. From December 2000 to 2004, he was an Adjunct Professor with the Faculty of Sciences and Engineering, Department of Electrical and Information Engineering, Laval University, Quebec, QC, Canada. From September 1996 to February 2001, he was on leave from Alexandria University. From September 1996 to January 1998, he was with the Electrical and Computer Engineering Department, International Islamic University Malaysia, and from February 1998 to February 2001, he was with the School of Electrical and Electronic Engineering, Nanyang Technological University, Singapore. His research interests include optical communications, optical CDMA, optical burst-switching, OFDM technology, and information theory.

Dr. Shalaby has served as a student branch counselor at Alexandria University, IEEE Alexandria and North Delta Subsection, from 2002 to 2006, and served as a chairman of the student activities committee of the IEEE Alexandria Subsection from 1995 to 1996. He received an SRC fellowship from 1987 to 1991 (Systems Research Center, Maryland); State Excellence Award in Engineering Sciences in 2007 (Academy of Scientific Research and Technology, Egypt); Shoman Prize for Young Arab Researchers in 2002 from The Abdul Hameed Shoman Foundation, Amman, Jordan; the State Incentive Award in Engineering Sciences both in 1995 and 2001 from the Academy of Scientific Research and Technology, Egypt; the University Excellence Award in 2009 from Alexandria University; and the University Incentive Award in 1996 from Alexandria University.

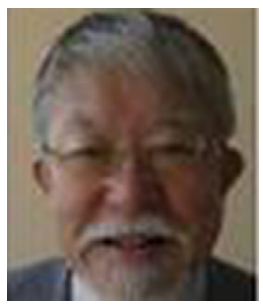

Zen-Ichiro Kawasaki received the B.S., M.S. and Dr.Eng degrees in Communication Engineering from Osaka University in 1973, 1975 and 1978 , respectively. In 1989 , he joined the Department of Electrical Engineering, Osaka University. At present, he is a professor of Electrical, Electronics and Information Engineering at Osaka University. $\mathrm{He}$ is also an advisor and dean at the Egypt-Japan University for Science and Technology, Alexandria, Egypt. His main research interests are in radar-based remote sensing, passive and active remote sensing of the atmosphere from space-born platforms, and atmospheric electricity. 